\title{
Vortex shedding from a spinning cylinder
}

\author{
F. Diaz, J. Gavalda, J. G. Kawall, ${ }^{\text {a) }}$ J. F. Keffer, ${ }^{\text {a) }}$ and F. Giralt \\ Departaments de Química Tècnica i Física, Facultat de Química, Universitat de Barcelona, Tarragona, \\ Catalunya, Spain
}

(Received 6 April 1983; accepted 30 August 1983)

\begin{abstract}
An experimental investigation of a two-dimensional turbulent wake behind a spinning cylinder at $\operatorname{Re}=9000$ is carried out to determine the influence of the rotation on the initial development of the flow. Spectral analysis of the velocity data measured in the near wake shows that for peripheral velocities up to the value of the free-stream velocity, a distinct Kármán vortex activity exists within the wake, whereas for greater peripheral velocities, the Kármán activity deteriorates and disappears for values in excess of twice the free-stream velocity.
\end{abstract}

\section{INTRODUCTION}

In recent years, there has been considerable interest in the role that coherent structures play within turbulent flows. The visualization study carried out by Papailiou and Lykoudis $^{1}$ in the wake of a circular cylinder has suggested that residual effects of the Kármán vortex street can persist in the fully developed turbulent wake. It is possible that these effects could be responsible for the triggering of the outer intermittent turbulent bulges of the wake and the existence of the coherent structures found by Grant $^{2}$ and Keffer. ${ }^{3}$ It should be noted, however, that Budny, Kawall, and Keffer ${ }^{4}$ have established that all Kármán vortex street activity effectively ceases to exist by about 65 cylinder diameters downstream. Subsequent investigations on the fully developed turbulent wake flow carried out by LaRue and Libby ${ }^{5}$ and Kawall and Keffer, ${ }^{6}$ and based on hot-wire anemometry and statistical processing of the signals, have shown that the turbulent bulges at the fully developed wake boundaries occur randomly. Moreover, results obtained by Kawall and Keffer ${ }^{7}$ indicate that the bulges are characterized by a frequency that is significantly lower than the Strouhal (or vortex shedding) frequency. This is consistent with the work of Cimbala, Nagib, and Roshko, ${ }^{8}$ who have found that largescale coherent structures with a characteristic wavenumber which is lower than that associated with the Kármán vortices evolve in the fully developed turbulent wake, and that these structures appear to result from instability of the mean velocity profiles of the developing flow.

In the light of the aforementioned findings, it is clear that a substantial amount of information on a wide variety of turbulent shear flows is needed before we can arrive at a comprehensive understanding of the mechanics of the coherent structures within the flow and of the role that these structures play in the turbulent transport processes. The present experimental investigation of the vortex shedding from a spinning cylinder has been undertaken in a new attempt to obtain some of this information. It may be remarked that this particular flow is of importance in connection with wind machines that utilize the Magnus effect to generate electrical power.

\footnotetext{
a) Department of Mechanical Engineering, University of Toronto, Toronto, Ontario, Canada
}

\section{EXPERIMENTAL DETAILS}

A definition sketch of the flow is shown in Fig. 1, where $x$ and $y$ are the streamwise and lateral coordinates, respectively. The flow was generated by means of a circular cylinder of diameter $D$ equal to $20 \mathrm{~mm}$, mounted horizontally in the center of a variable-speed, open-circuit wind tunnel. This tunnel, which is located in the Departament de Química Tècnica, Universitat de Barcelona, Tarragona (UBT), is fully described by Diaz. ${ }^{9}$ The cylinder was supported in bearings attached to the tunnel walls, with one end of the cylinder protruding through the tunnel walls and connected to a variable-speed motor so that it could be rotated at selected peri-

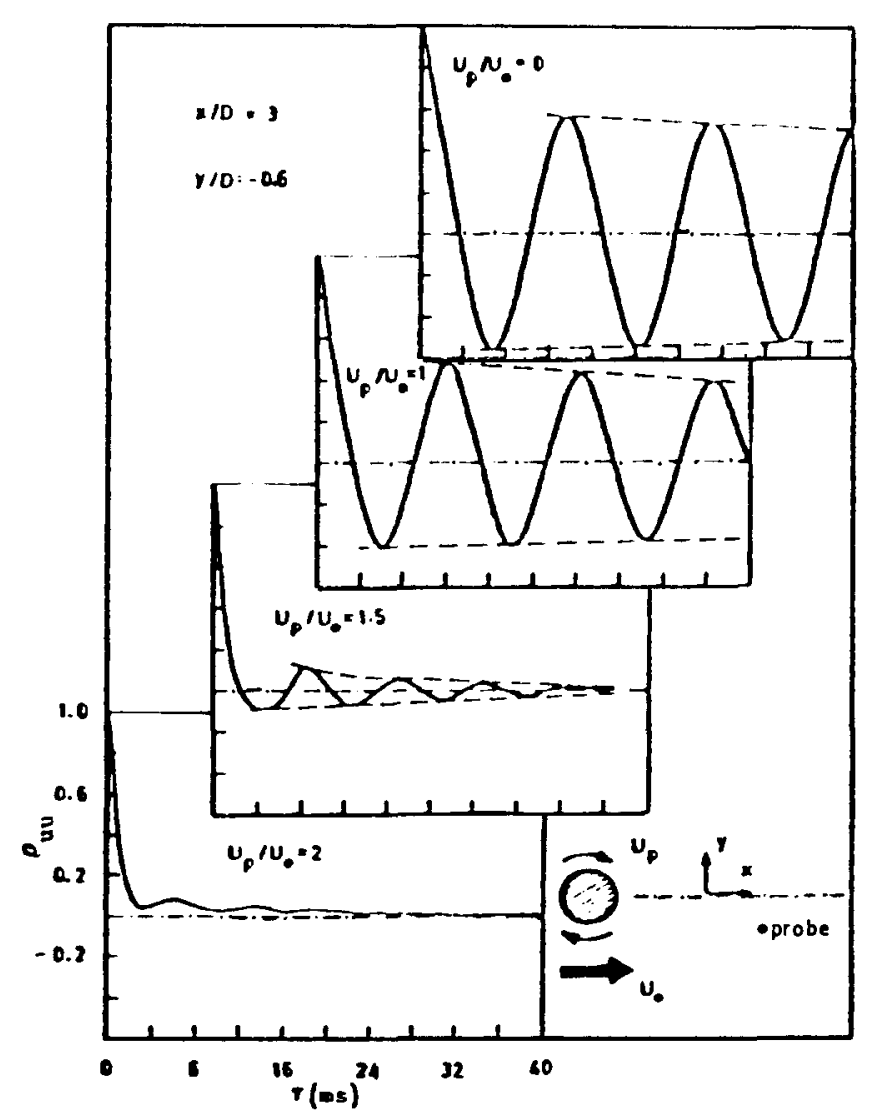

FIG. 1. Streamwise velocity autocorrelations at $x / D=3$ and $y /$ $D=-0.6$ 
pheral speeds $U_{p}$ up to 2.5 times the free-stream velocity, $U_{0}$. The tunnel was operated at a speed, $U_{0}$, of $7 \mathrm{msec}^{-1}$, which produced a cylinder Reynolds number of about 9000 . The length-to-diameter ratio of the cylinder was in excess of 50, so that the statistical properties of the flow were effectively functions of $x$ and $y$ only (i.e., the wake was two dimensional).

Data were obtained in the vertical center plane of the tunnel at a number of downstream locations corresponding to $x / D$ between 3 and 10 . Analog voltage signals related to the instantaneous streamwise and lateral velocities of the flow were obtained by means of DISA $55 \mathrm{M}$ constant-temperature anemometers, in conjunction with two normal hotwire probes which were oriented so that their wires were at $90^{\circ}$ to $U_{0}$, and a $45^{\circ} \mathrm{X}$-wire probe which was orientated so that its wires were at $45^{\circ}$ to $U_{0}$. The hot-wire signals were recorded on magnetic tape at UBT, and subsequently lowpass filtered and digitally sampled at the University of Toronto. The low-pass filter value and sampling rate used were 2 $\mathrm{kHz}$ and 7042 points per second, respectively. The digital data were stored on magnetic tapes and processed on an IBM 3033 computer. Preliminary measurements ${ }^{9}$ indicated that the curvature of the mean streamlines of the flow that resulted when $U_{p} / U_{0}$ was significantly greater than zero was negligibly small for $x / D$ in excess of about 2.5 , signifying that the mean direction of the flow was essentially the same as that of the free stream for all the downstream locations examined herein. Accordingly, it was possible to determine the instantaneous streamwise velocities pertaining to these locations from the normal hot-wire probe signals, and instantaneous streamwise and lateral velocities from the $X$ wire probe signals.

\section{ANALYTICAL CONSIDERATIONS}

The autocorrelation function of a signal $\alpha(t)$ with zero mean, obtained at a given point in a turbulent flow, is defined as

$$
\begin{aligned}
\rho_{\alpha \alpha}(\tau) & =\overline{\alpha(t) \alpha(t+\tau)} / \overline{\alpha^{2}} \\
& =\frac{\lim _{T \rightarrow \infty}(1 / T) \int_{0}^{T} \alpha(t) \alpha(t+\tau) d t}{\overline{\alpha^{2}}},
\end{aligned}
$$

where $\tau$ is a time lag and $\overline{\alpha^{2}}$ is the variance of the signal.

The one-dimensional spectrum of $\alpha(t)$, i.e., the Fourier transform of $\overline{\alpha^{2}} \rho_{\alpha \alpha}(\tau)$, is given by

$$
E_{\alpha \alpha}(n)=2 \int_{-\infty}^{\infty} \rho_{\alpha \alpha}(\tau) \exp (-i 2 \pi n \tau) d \tau,
$$

where $n$ is a frequency such that $n>0$.

The coherence between any given Fourier components of two signals $\alpha_{1}(t)$ and $\alpha_{2}(t)$ with zero mean, measured at two points separated by a distance $s$, is described by the coherence function

$$
\Gamma_{\alpha_{1} \alpha_{2}}(n, s)=\frac{C_{\alpha_{1} \alpha_{2}}^{2}(n, s)+Q_{\alpha_{1} \alpha_{2}}^{2}(n, s)}{E_{\alpha_{1} \alpha_{1}}(n) E_{\alpha_{2} \alpha_{2}}(n)}
$$

The functions $C_{\alpha_{1} \alpha_{2}}$ and $Q_{\alpha_{1} \alpha_{2}}$, which are referred to as the cospectrum and quadrature spectrum, respectively, are the real and imaginary components of the cross spectrum of $\alpha_{1}$ and $\alpha_{2}$.

\section{RESULTS AND DISCUSSION}

Normalized autocorrelation functions of the streamwise velocity component measured at $x / D=3$ and $y /$ $D=-0.6$ are presented in Fig. 1 for different values of $U_{p} / U_{0}$. These results show that Kármán vortex street activity has a dominant role in the near wake for peripheral speeds up to the free-stream velocity. As can be seen, the amplitude of the oscillations decay with increasing $\tau$, indicating that vortex shedding from a rotating circular cylinder is not a strictly periodic process but a randomly modulated one, as established by Budny et al. ${ }^{4}$ and Keffer and Kawall ${ }^{10}$ for a stationary cylinder situation. The oscillatory behavior of $\rho_{u u}(\tau)$ is rapidly damped when $U_{p}$ exceeds $U_{0}$, and for $U_{p} / U_{0}>2, \rho_{u u}(\tau)$ decays monotonically and rapidly to zero. From this, it can be concluded that the micro- and macroscales of the flow in the near wake decrease as the peripheral velocity of the cylinder increases. It should be mentioned that the autocorrelation functions pertaining to $U_{p} / U_{0}>2.5$ showed a behavior typical of homogeneous turbulence.

The results presented in Fig. 1 serve to demonstrate that Kármán vortex street activity diminishes significantly when $U_{p}>U_{0}$. Furthermore, since attenuation of the periodic behavior of $\rho_{u u}(\tau)$ increases markedly when $U_{p} / U_{0}$ increases from 1.0 to 1.5 , these results imply that a fundamental change occurs in the process that governs the formation of the Kármán vortices when $U_{p}$ increases beyond $U_{0}$. The decay of the Kármán street and the increase in the random modulation of the shedding process with rotational speed has to be linked to the displacement of the stagnation points and to the thickening of the layer of rotating fluid near the cylinder surface with rotation, which cause separation to occur progressively away from the surface of the cylinder and under different lateral velocity gradients in the regions above and below the cylinder. We note that these findings are consistent with data obtained by Díaz, Gavaldà, Kawall, and Giralt, " which establish that the wake width decreases significantly once the peripheral velocity exceeds the freestream velocity.

The effects of the cylinder rotation on the kinetic energy associated with the longitudinal fluctuating velocity at $x$ / $D=3$ and $y / D=+0.6$ and -0.6 are illustrated in Figs. 2 and 3. At $y / D=-0.6$, a distinct frequency-centered activity, i.e., a narrow-band peak, exists in the autospectrum, $E_{u u}(n)$, for $0<U_{p} / U_{0}<1.0$. The nominal frequency at which this spectral peak occurs corresponds to the Strouhal frequency, $n_{s}$, of $76 \mathrm{~Hz}$ (which pertains to $U_{p} / U_{0}=0$ ), and shifts by a small but significant amount when $U_{p} / U_{0}$ increases. It is apparent from these results that the third-harmonic $\left(3 n_{s}\right)$ Fourier component is more energetic than the second-harmonic one, signifying that the longitudinal velocity signals at this laterol location, $y / D=-0.6$, in the near wake are strongly skewed. For $U_{p} / U_{0}$ between 1.0 and 1.5 , there is a substantial decrease in the energy associated with the Kármán vortices, and the spectral peak becomes broadband in nature. This establishes that the (normal) random modulation of the shedding process ${ }^{4.10}$ increases sharply 


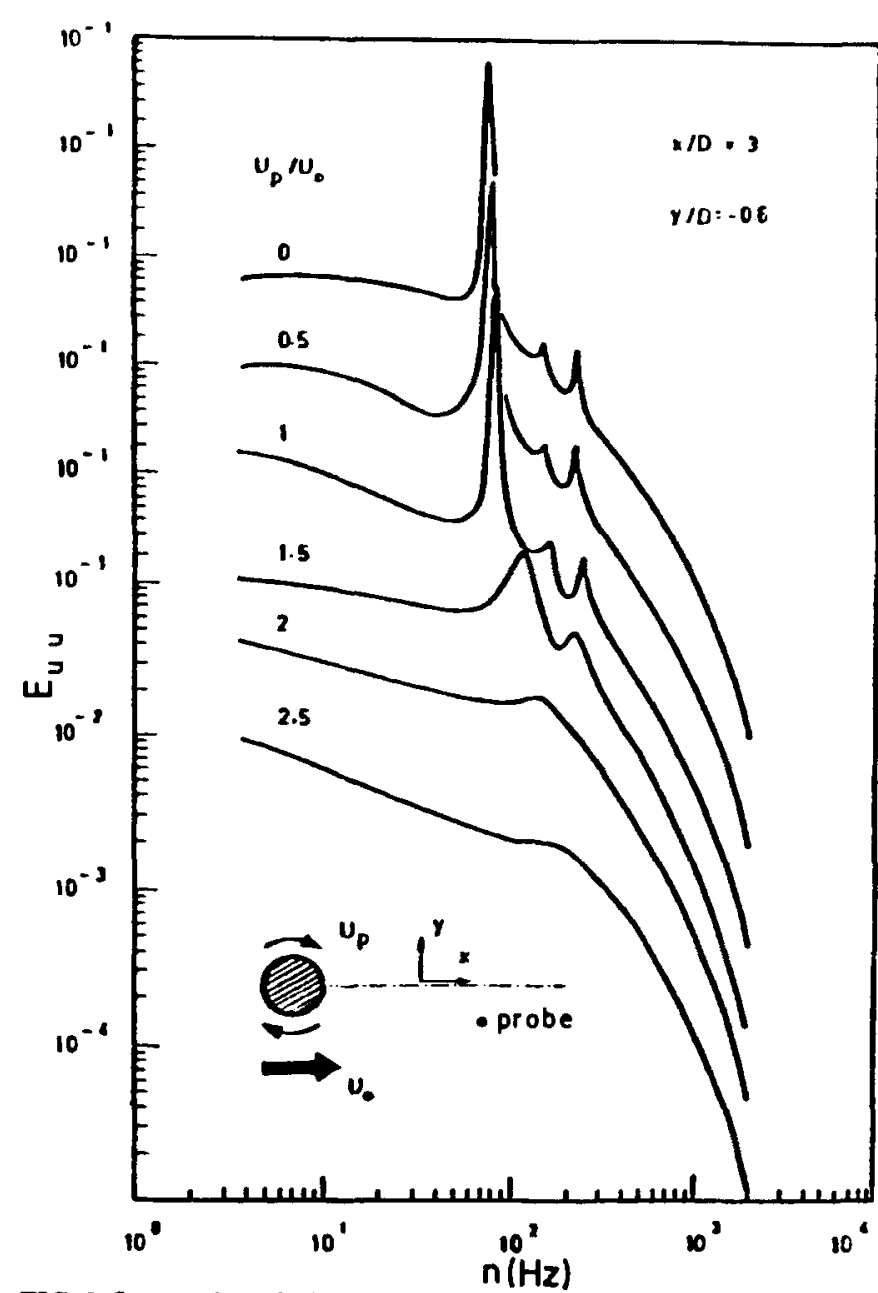

FIG. 2. Streamwise velocity autospectra at $x / D=3$ and $y / D=-0.6$.

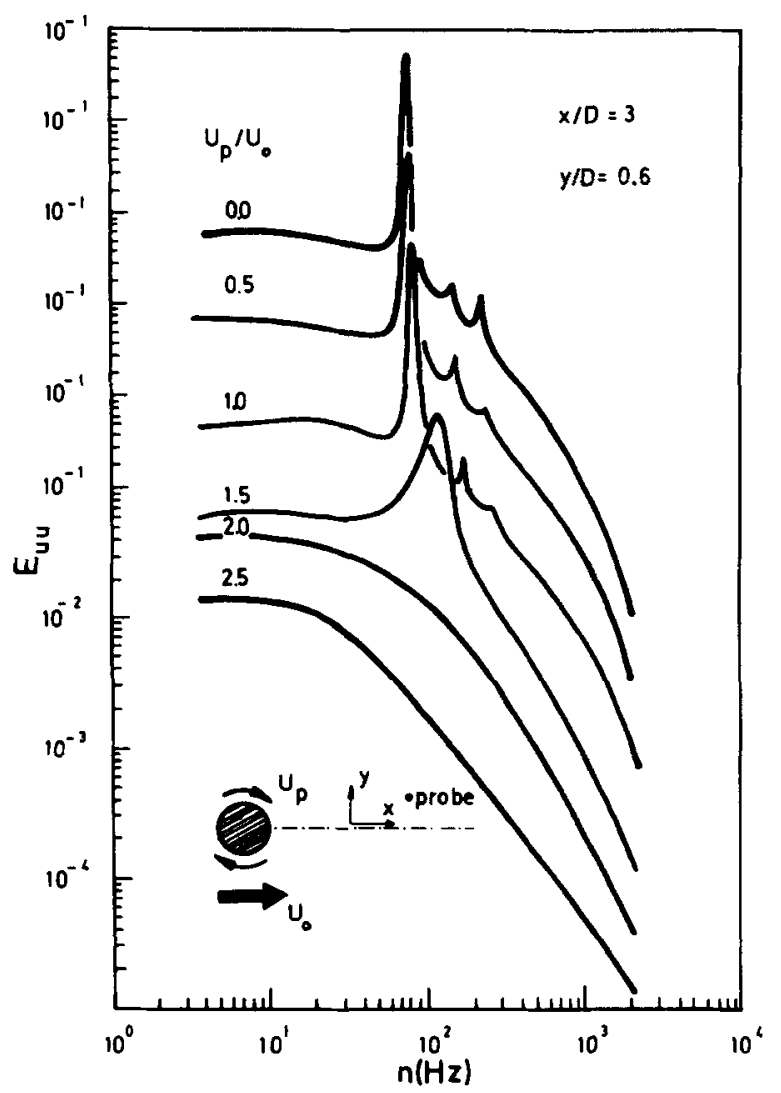

FIG. 3. Streamwise velocity autospectra at $x / D=3$ and $y / D=0.6$. when $U_{p} / U_{0}$ is much in excess of 1.0: vortex shedding still takes place, but a distinct shedding frequency no longer exists. When $U_{p} / U_{0}$ is greater than about 2.0 , this process becomes completely random; moreover, when $U_{p} / U_{0}$ exceeds this latter value and the layer of fluid dragged around with the rotating cylinder is sufficiently thick, vortex shedding as such ceases. The autospectrum data for $y / D=+0.6$ are seen to be consistent with those for $y / D=-0.6$. The slight differences between the two sets of data are consequences of the lateral deflection and compression of the wake caused by cylinder rotation. The $E_{u u}(n)$ data determined at $y / D=0$, which are presented in Fig. 4, display similar trends except in the case of $U_{p} / U_{0}=0$, for which a broadband spectral peak centered at $2 n_{S}$ is evident. The location of this peak reflects the fact that when $U_{p} / U_{0}=0$, a probe located at $y / D=0$ senses the passage of the Kármán vortices shed from both the upper and lower parts of the cylinder; and the broadband nature of the peak stems from the fact that the fluid motion in the outer regions of these vortices is diffuse and gives rise to polychomatic signals.

Figure 5 shows the coherence functions of longitudinal velocity components, $u_{1}$ and $u_{2}$, measured at $x / D=3$ and $y_{1} / D=+0.6$ and $y_{2} / D=-0.6$, pertaining to various rotational speeds. (It should be mentioned here that for each of the rotational speeds employed in the present work, detailed lateral hot-wire traverses of the flow were carried out at $x$ / $D=3$, and it was established from an examination of traces

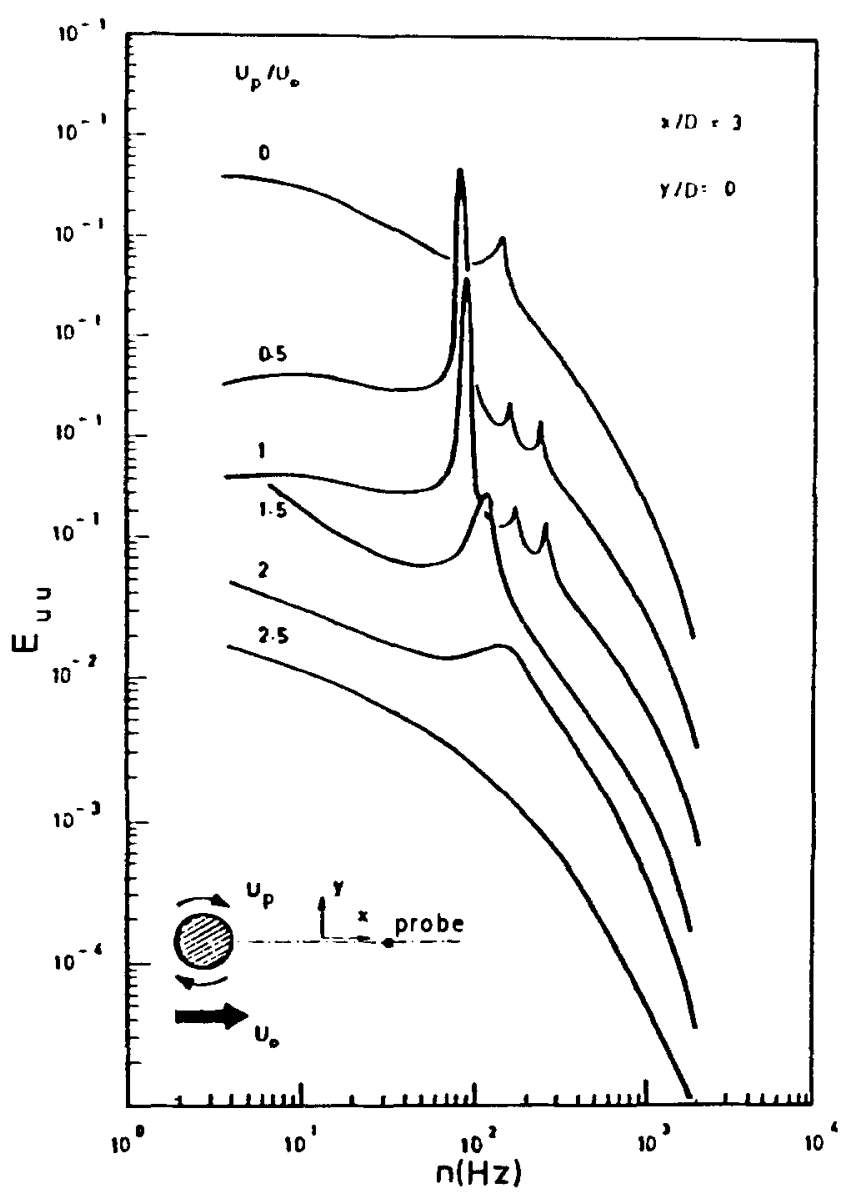

FIG. 4. Streamwise velocity autospectra at $x / D=3$ and $y / D=0.0$. 


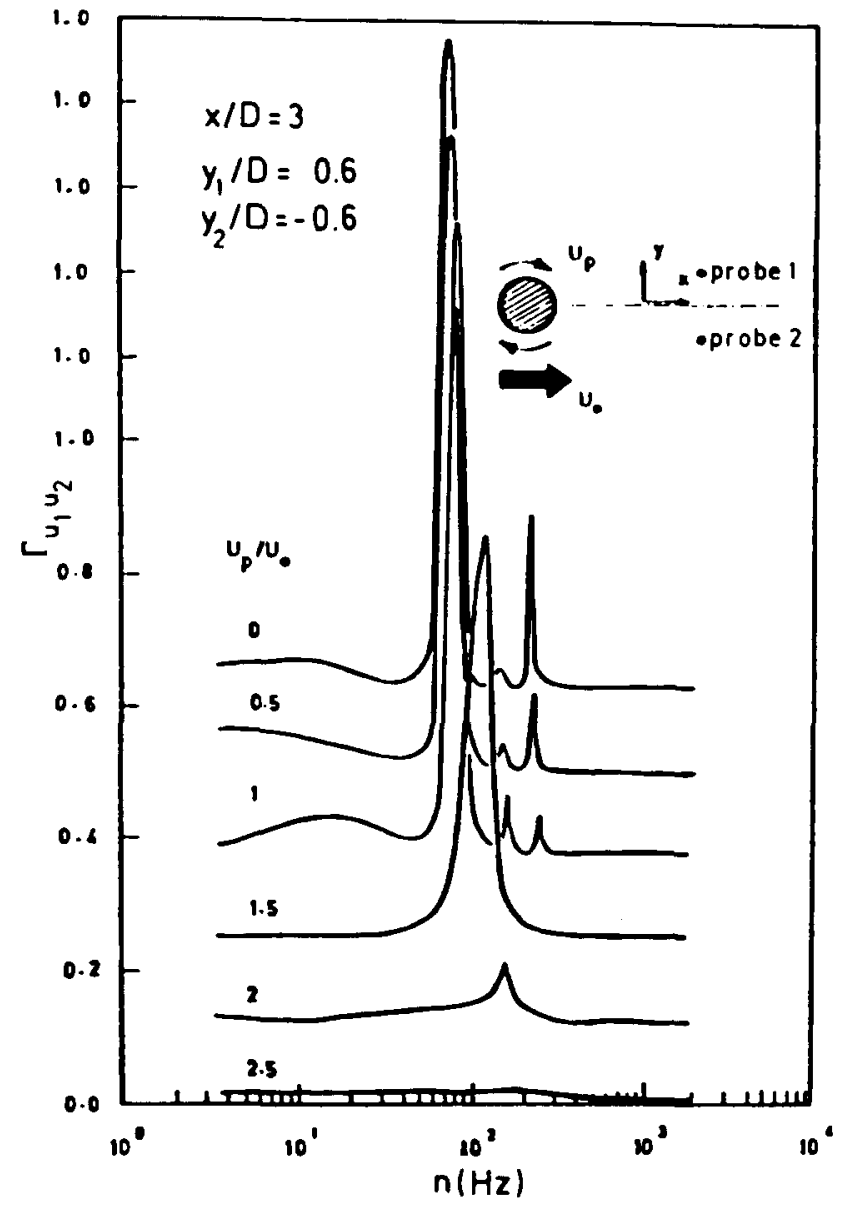

FIG. 5. Two-point coherence functions at $x / D=3$ and $y / D=0.6$ and -0.6 .

of the associated hot-wire signals that a probe located at $y$ / $D=-0.6$ would be located within the wake either continuously or intermittently depending upon the particular rotational speed involved.) As can be seen, for $U_{p} / U_{0}<1, u_{1}$ and $u_{2}$ are highly coherent over a relatively narrow frequency range centered at the Strouhal frequency $n_{s}$. Moreover, the skewed character of the velocity signals, mentioned above, can be inferred from these results as well, since the degree of coherence is greater at $3 n_{s}$ than it is at $2 n_{s}$, for $U_{p} / U_{0}<1.0$. As $U_{p} / U_{0}$ increases from 1.0 to 2.0 , because of the rapid randomization of the shedding process, the nominal frequency at which the coherence function displays a dominant peak increases by a noticeable amount, the coherence level decreases significantly, and the peak becomes broadband. For $U_{p} / U_{0}$ much in excess of 2.0 , the coherence between $u_{1}$ and $u_{2}$ is effectively zero at all frequencies. The coherence functions of $u_{1}$ and $u_{2}$ measured at $y_{1} / D=0$ and $y_{2} /$ $D=-0.6$ that are presented in Fig. 6 are seen to exhibit much the same traits except with respect to $U_{\rho} / U_{0} \geqslant 2.0$. For this condition, $u_{1}$ and $u_{2}$ are slightly coherent over a limited range of relatively low frequencies $\left(<n_{S}\right)$.

On the basis of the autospectral results (Figs. 2-4) and the coherence function results (Figs. 5 and 6), it can be concluded that once $U_{p} / U_{0}>2.0$, no coherent structures associated with Kármán vortex activity exist within the near wake, and hence, the latter can be considered fully turbulent, with its fluctuation kinetic energy distributed over all rel-

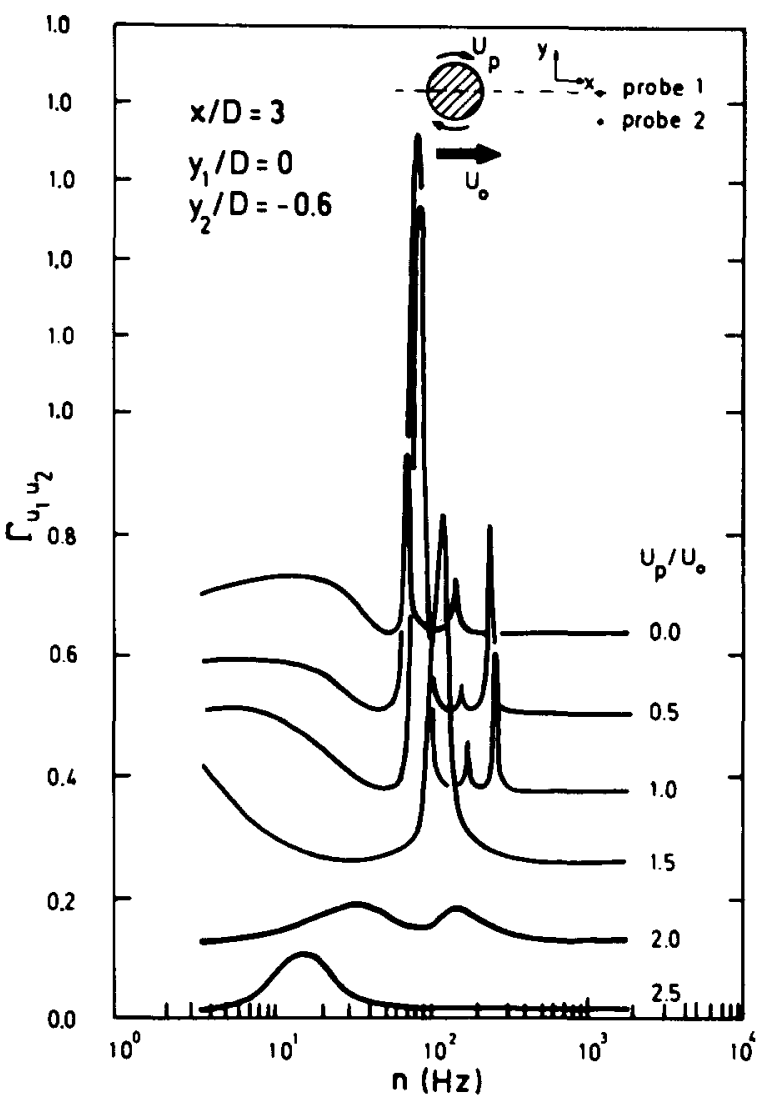

FIG. 6. Two-point coherence functions at $x / D=3$ and $y / D=0.0$ and -0.6 .

evant frequencies (see Figs. 2-4). We speculate that the "lowfrequency" band-limited coherence observed in Fig. 6 for $U_{p} / U_{0}>2.0$, which is clearly not a result of Kármán vortex street activity, could be due to longitudinal Goertler vortices, typical of boundary layers over concave surfaces, coupled with Taylor ring vortices formed around the cylinder when the peripheral velocity is sufficiently high with respect to the free-stream velocity to produce a significant layer of entrained rotating fluid and hence, generate centrifugal instabilities, similar to those associated with two concentric

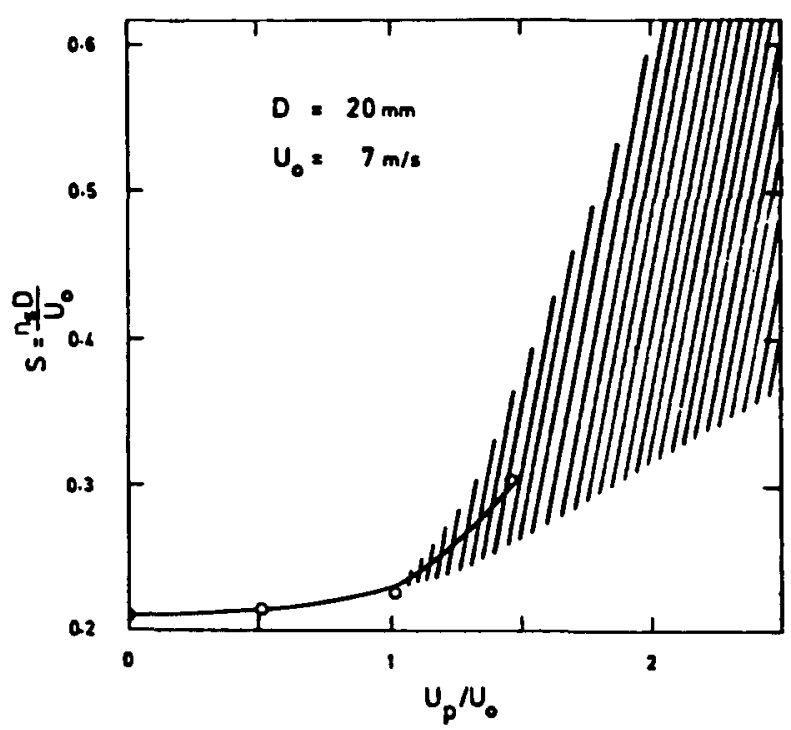

FIG. 7. Variation of the Strouhal number with peripheral velocity. 


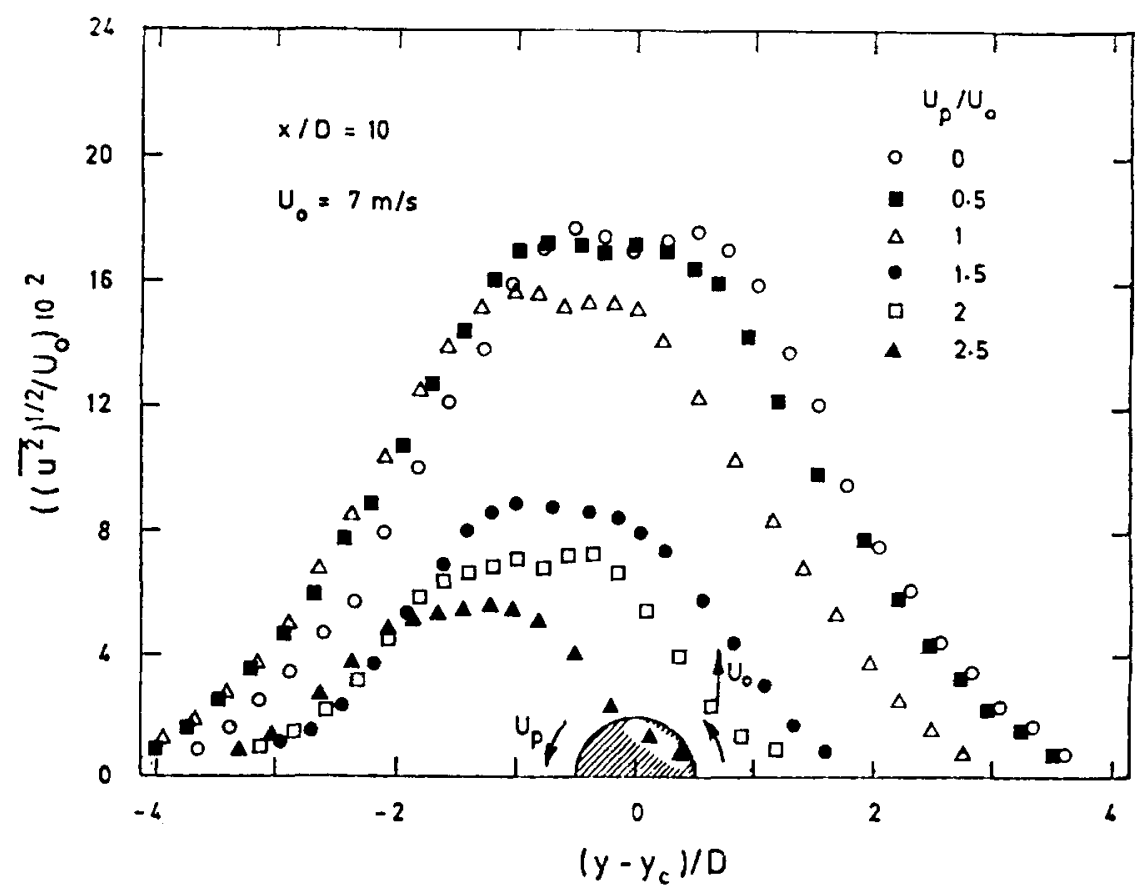

FIG. 8. Lateral distributions of the streamwise turbulence intensitieis at $x / D=10$.

rotating cylinders. Preliminary flow visualization experiments conducted at UBT seem to support our conjecture, and steps are being taken to refine these experiments in order to obtain definitive information on this aspect. It should be pointed out that the aforementioned "low-frequency" peak is not present in the coherence functions pertaining to $y_{1} /$ $D=+0.6$ and $y_{2} / D=-0.6$ (Fig. 5) because the wake is increasingly deflected in the negative $y$ direction as the rotational speed of the cylinder increases, and, as a result, when $U_{\rho} / U_{0}$ exceeds 1.5 (and Kármán vortex activity ceases to exist), the probe located at $y_{1} / D=+0.6$ is, on the average, immersed in the potential flow while the probe located at $y_{2} / D=-0.6$ is immersed in the wake flow.

The variation of the Strouhal number, $S\left(=n_{S} D / U_{0}\right)$, with $U_{p} / U_{0}$ is shown in Fig. 7. It can be seen that $S$ is about 0.21 for $U_{p} / U_{0}=0$ - as it should be with respect to a stationary cylinder situation involving a well-defined Kármán vortex street-and that it increases gradually as $U_{p}$ approaches $U_{0}$. For $U_{p} / U_{0}$ much in excess of 1.0 , a specific Strouhal number cannot be defined, since the shedding process then becomes rapidly more random and no definite shedding frequency can be inferred from the autospectral results (see Figs. 2 and 3).

Typical lateral distributions of the rms streamwise and

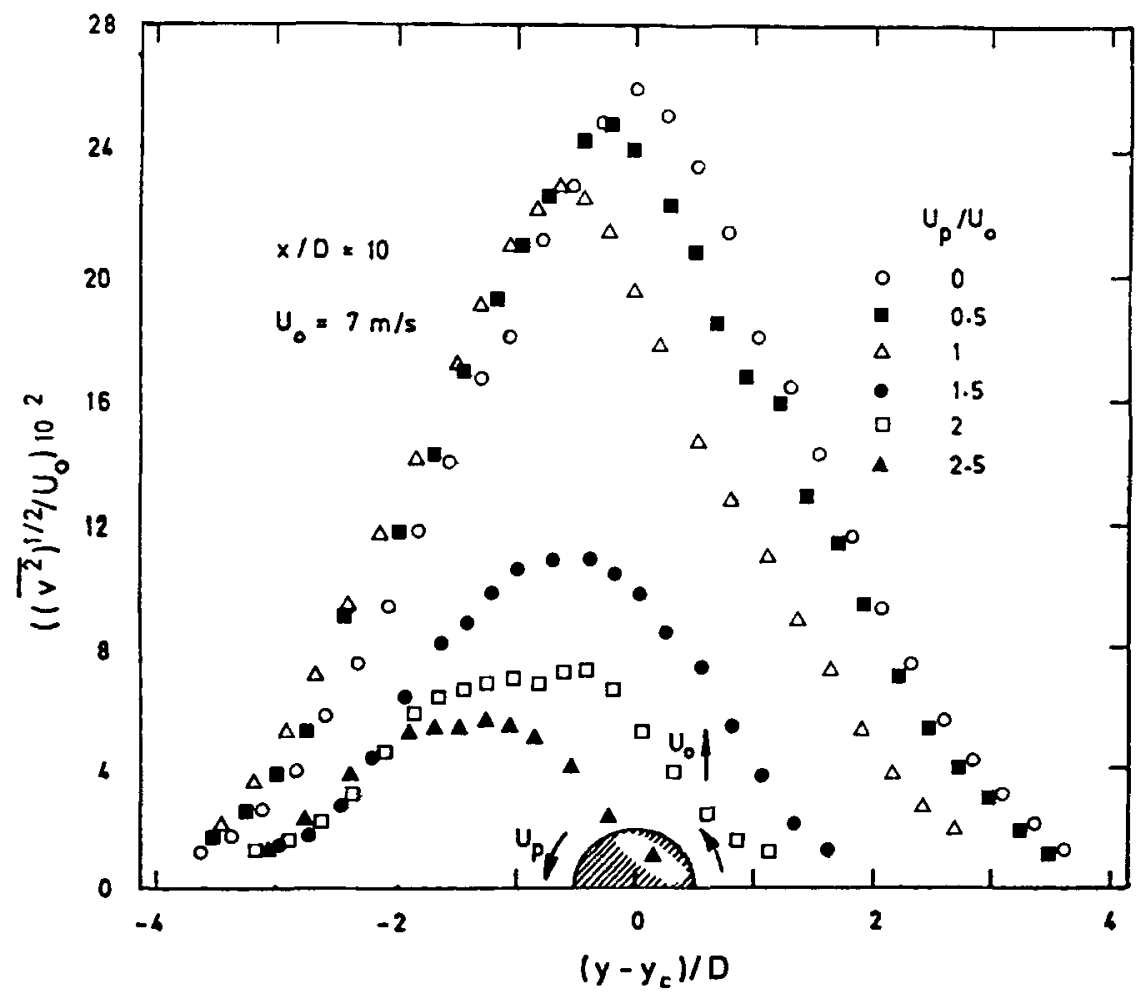

FIG. 9. Lateral distributions of the lateral turbulence intensities at $x / D=10$. 


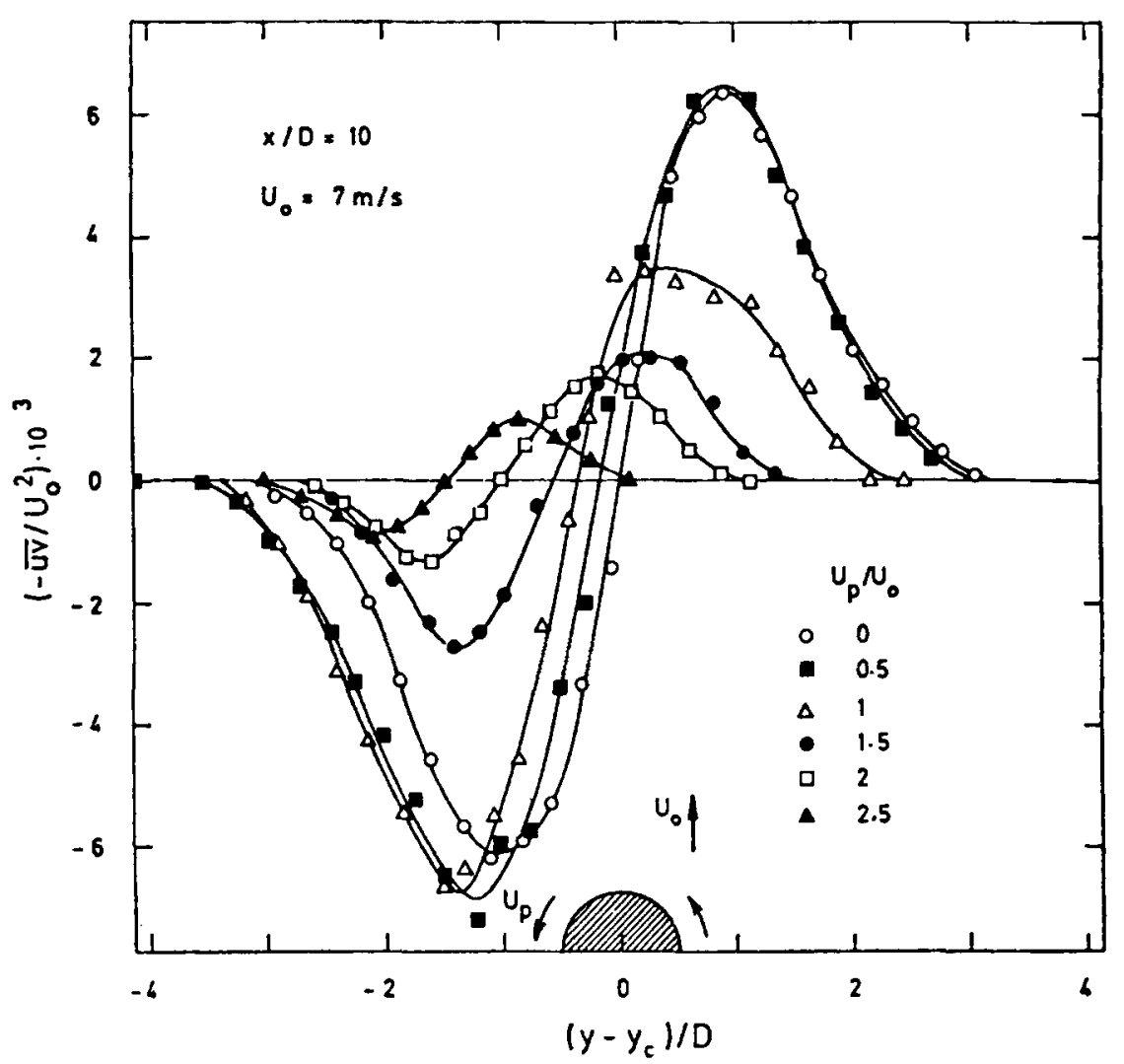

FIG. 10. Lateral distributions of the Reynolds shear stresses at $x / D=10$. lateral velocity components $u^{\prime}$ and $v^{\prime}$, and of the Reynolds shear shress $\overline{u v}$ measured at $x / D=10$ are illustrated in Figs. 8-10 for different rotational speeds. Note that, as expected, $v^{\prime}$ is greater than $u^{\prime}$ in this initial-wake region where Kármán vortex activity (pseudosolid rotation) dominates the flow for $U_{p} / U_{0}<1.0$ and lateral wake growth is important. The variance and correlation of the fluctuating quantities plotted in these figures reveal that as $U_{p} / U_{0}$ increases (i) the fluctuating field of the wake is damped, especially for $U_{p} / U_{0}>1.0$, (ii) the wake is increasingly displaced in the negative lateral direction, and (iii) the width of the wake decreases, particularly for $U_{p} / U_{0}>1.0$. These results, which are consistent with the spectral data obtained at $x / D=3$, indicate that it is possible to distinguish two different flow regimes in the wake behind a spinning cylinder: one associated with $U_{p} / U_{0}<1.0$, and one associated with $U_{p} / U_{0}>1.0$. In the case of the former regime, the wake flow is dominated, in the early stages of its evolution, by the presence of welldefined, highly energetic Kármán vortices. In the case of the latter regime, these structures become progressively diffuse as $U_{p} / U_{0}$ increases and eventually cease to exist for $U_{p} / U_{0} \geqslant 2.0$.

The aforementioned regimes are evidently related to the thickness of the layer of fluid dragged around with the spinning cylinder, and therefore, to the velocity profiles in the immediate vicinity of the top and bottom of the cylinder. These profiles are identical when the cylinder is stationary, corresponding to a symmetrical flow configuration. But, as depicted in Fig. 11, they become increasingly dissimilar as the peripheral velocity increases, and are quite different once this velocity exceeds the free-stream velocity, the flow con- figuration then being completely asymmetrical. Moreover, for $U_{p} / U_{0}>0$, whereas the direction of the fluid velocity remains the same in the flow region above the top of the cylinder, it reverses in the flow region below the bottom of the cylinder-the implication here being that relatively "high momentum transfer" conditions prevail in the region below the cylinder surface. In consequence, for $0<U_{p} / U_{0} \leqslant 1.0$, boundary layer separation and vortex shedding are less favored from the upper cylinder surface than they are from the lower cylinder surface, and hence, the wake is deflected in the negative $y$ direction. For $U_{p} / U_{0}>1.0$, vortex shedding is increasingly inhibited, and this results in the previously discussed reduction of the fluctuation kinetic energy of the wake, redistribution of this kin-

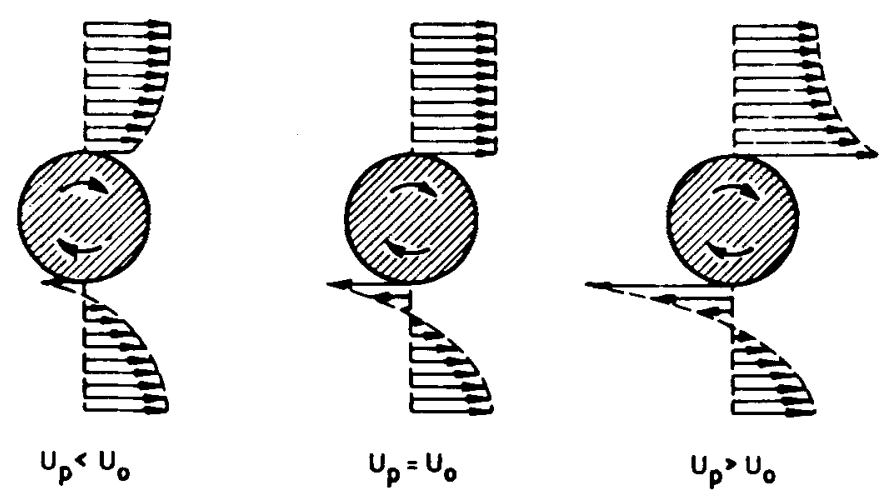

FIG. 11. Sketch of the lateral velocity profiles near the top and bottom of a spinning cylinder. 
etic energy over all relevant frequencies, and reduction of the wake width.

\section{SUMMARY AND CONCLUDING REMARKS}

The results obtained in the present study establish that shedding of Kármán vortices in a cylinder-generated wake is modified by rotation of the cylinder. It is possible to distinguish two different flow regimes under these circumstances. One regime is associated with $U_{p} / U_{0} \leqslant 1.0$ and involves a "quasiconventional" cylinder-generated wake; the other regime is associated with $U_{p} / U_{0}>1$ and involves a flow without Kármán vortex street activity in its near region. Correlation and spectral analysis of longitudinal velocity signals demonstrate that for $U_{p} / U_{0} \leqslant 1$, distinct Kármán vortex street activity exists in the flow and the frequency characterizing this activity increases from the conventional Strouhal frequency $\left(=0.21 D / U_{0}\right)$ by a small but significant amount as $U_{p}$ increases; for $U_{p} / U_{0}>1$, this activity diminishes and eventually disappears when $U_{p} / U_{0}>2$, signifying that the wake is then fully turbulent from its inception.

\section{ACKNOWLEDGMENTS}

This research was financially supported by CIRIT (Generalitat de Catalunya), DOW CHEMICAL Ibérica
(Tarragona, Spain), and the Natural Sciences and Engineering Research Council of Canada under Grant No. A-2746. Technical support was provided by TAQSA (Tarragona, Spain).

'D. D. Papailiou and P. S. Lykoudis, J. Fluid Mech. 62, 11 (1974).

${ }^{2}$ H. L. Grant, J. Fluid Mech. 4, 149 (1958).

${ }^{3}$ J. F. Keffer, J. Fluid Mech. 22, 135 (1965).

${ }^{4}$ R. S. Budny, J. G. Kawall, and J. F. Keffer, in Proceedings of the 2nd Symposium on Turbulent Shear Flows, London, England (Imperial College, London, 1979), p. 7.20.

${ }^{5}$ J. C. LaRue and P. A. Libby, Phys. Fluids 19, 1864 (1976).

6J. G. Kawall and J. F. Keffer, Phys. Fluids 22, 31 (1979).

${ }^{7}$ J. G. Kawall and J. F. Keffer, in Turbulent Shear Flows, edited by L. J. S. Bradbury, F. Durst, B. E. Launder, F. W. Schmidt, and J. H. Whitelaw (Springer-Verlag, New York, 1982), Vol. 3, p. 132.

${ }^{8}$ J. Cimbala, H. Nagib, and A. Roshko, Bull. Am. Phys. Soc. Ser. II 26 , 1256 (1981).

${ }^{9} \mathrm{~F}$. Díaz, Ph.D. thesis, Universitat de Barcelona, 1982.

${ }^{10} \mathrm{~J}$. F. Keffer and J. G. Kawall, presented at the AIAA 13th Fluid and Plasma Dynamics Conference, Snowmass, Colorado, 1980, Paper 80. 1337.

"F. Díaz, J. Gavaldà, J. G. Kawall, and F. Giralt, in Structure of Complex Turubulent Shear Flow, edited by R. Dumas and L. Fulachier (SpringerVerlag, New York, 1983), p. 175. 